worked before delivery. 584 pregnant workers were included in this study, yielding the cover rate $82.60 \%$. The analysis was performed by percentage and regression. The result found that the proportion between returned to work and non-returned to work 6 months after delivery was $4: 1$. There are three general characteristic factors with statistical significance associated with returning to work, which include age (p-value 0.002), number of pregnancy ( $\mathrm{p}$-value $<0.001$ ), and source of income ( $\mathrm{p}$-value $<0.001)$. In addition, there are eight occupational factors with statistical significance associated with returning to work consisting of occupation ( $\mathrm{p}$-value 0.03), employment status ( $\mathrm{p}$-value 0.03 ), working sectors ( $\mathrm{p}$-value $<0.001$ ), fulltime or part-time (p-value <0.001), work posture such as standing ( $\mathrm{p}$-value $<0.001$ ), sitting (p-value $<0.001$ ), walking ( $\mathrm{p}$-value 0.048 ), and poor ergonomic posture (p-value 0.02). Furthermore, there is one maternal factors with statistical significance associated with returning to work, which was postpartum hematoma ( $\mathrm{p}$-value 0.003). However, there are no infant factors with statistical significance associated with returning to work. In conclusion, this study found factors related to returning to work. The occupational health provider should include these factors for holistic care in this working population.

\section{EFFECTS OF ROTATING-SHIFT WORK ON FEMALE SEXUAL FUNCTION IN NURSE - TWO HOSPITALS STUDY}

R Y Chen, Yeh, Lin. Taipei Medical University, Taipei, Taiwan

\subsection{6/oemed-2013-101717.98}

Objective The main purpose of this study is to examine the impact of night-shift task on the female sexual function, such as sexual desire, arousal, lubrication, orgasm, satisfaction and sexual pain among the female nurses.

Method The study subjects were female nurses who were 20-49 years old and worked in Changhua and Chang Bing ShowChwan Memorial Hospital. All the study nurses were asked by questionnaire about their time-shift working status, quality of sleep, health status and sexual function. Data were analysed by Statistical Package for Social Science (SPSS).

Results Compared to day-shift nurses, night-shift nurses had a higher risk to have poor sleep quality and self-report health status $(\mathrm{p}<0.05)$. The averaged female sexual function index among the study nurses was $56.42 \pm 9.12 \square$ There was $68.85 \%$ of the study nurses had at least one kind of female sexual dysfunction. The increased age, body mass index, poor sleep quality, and sexual dysfunction of partner were the risk factors of female sexual dysfunction $(\mathrm{P}<0.05)$. The odd ratio of sexual dysfunction of partner was $4.997(95 \% \mathrm{CI}=-1.341 \sim-8.654 ; \mathrm{P}=$ $0.008)$.

Conclusion Night-shift task can cause female nurses to have poor sleep quality, and self reported health status. The female sexual function was also influence by different work and different shift way. The female sexual function was also influence by age, body mass index, partner sexual function, sleep quality and health status.

\section{Session: M. Cancer epidemiology II}

\section{ASSOCIATIONS OF POLYMORPHISMS IN CIRCADIAN GENES, SHIFT WORK AND BREAST CANCER IN THE GERMAN GENICA STUDY}

${ }^{1} S$ Rabstein, ${ }^{2} \mathrm{~V}$ Harth, ${ }^{1} \mathrm{~B}$ Pesch, ${ }^{3} \mathrm{C}$ Justenhoven, ${ }^{4} \mathrm{C}$ Baisch, ${ }^{4} \mathrm{M}$ Schiffermann, ${ }^{1} \mathrm{E}$ Heinze, ${ }^{3} \mathrm{H}$ Brauch, ${ }^{5} \mathrm{U}$ Hamann, ${ }^{4} \mathrm{Y}$ Ko, ${ }^{1} \mathrm{~T}$ Brüning. ${ }^{1}$ Institute for Prevention and Occupational Medicine (IPA), Bochum, Germany; ${ }^{2}$ Institute and Outpatient Clinic of Occupational Medicine (IAUP), Homburg, Germany, Germany; ${ }^{3}$ Dr. Margarete Fischer-Bosch-Institute of Clinical Pharmacology, Stuttgart, Germany; ${ }^{4}$ Department of Internal Medicine, Johanniter-Krankenhaus Bonn, Bonn, Germany; ${ }^{5}$ Molecular Genetics of Breast Cancer, Deutsches Krebsforschungszentrum (DKFZ), Heidelberg, Germany

\subsection{6/oemed-2013-101717.99}

Objectives Recently, the role of night-shift work in breast cancer development has been intensively discussed. Common variants in genes that regulate the circadian system may modify the observed risks of shift work. Here, we hypothesised that circadian genes influence breast cancer risk and may modify the risk of night shift work to develop breast cancer.

Material and Methods The population based case-control study Gene-Environment Interaction and Breast Cancer (GENICA) was conducted in the Greater Region of Bonn, Germany. Shift work and detailed shift work characteristics were assessed in subsequent telephone interviews. Thirteen polymorphisms in circadian genes AANAT, ARNTL, CLOCK, CRY2, MTNR1B, NPAS2, PER2, UGT1A, UGT1A6, UGT2B7, and UGT2B15 were genotyped. Associations between polymorphisms, shift work and breast cancer could be investigated for 1022 controls and 1014 cases. Risk estimates were calculated as odds ratios (ORs) with 95\% confidence intervals (CIs) conditional on age and adjusted for hormone replacement therapy, number of mammograms and familial breast cancer. Test for interactions as well as methods for Multifactor Dimensionality Reduction will be presented.

Results First results indicate elevated risk estimates for polymorphism rs8150 of gene AANAT (GC + CC vs. GG: OR 1.17; 95\% CI 1.01-1.36). In women that ever worked in shift for at least one year we found an elevated risk estimate for polymorphism rs10462028 in CLOCK gene (OR 3.53; 95\% CI 1.0911.42).

Discussion Our study suggests that polymorphisms in circadian genes may be associated with breast cancer and may also modify the risks of shift work for breast cancer. However, the results are limited by low prevalence of night work and variant genotypes. Therefore a pooling of studies would improve the statistical power to analyse the influence of circadian genes in breast cancer development.

\section{IMPACT OF SMOKING ON TOTAL AND MAJOR CAUSES OF MORTALITY AMONG CHINESE SILICOTICS IN HONG KONG: EFFECT MODIFIER OR CONFOUNDER?}

${ }^{1} \mathrm{Yu},{ }^{2} \mathrm{Tse},{ }^{2} \mathrm{Qiu},{ }^{3}$ Leung. ${ }^{1} \mathrm{NT}$, Hongkong; ${ }^{2}$ The Chinese University of Hong Kong, Hong Kong SAR, Hongkong; 'Pneumoconiosis Clinic, Tuberculosis and Chest Service, Department of Health, Hong Kong SAR, Hongkong

\subsection{6/oemed-2013-101717.100}

Objectives Whether smoking being an effect modifier or confounder in increased total mortality and mortality from major causes has never been formally evaluated in occupational cohort studies and this lack of knowledge was addressed in a cohort of Chinese workers with silicosis.

Methods All workers with silicosis in Hong Kong diagnosed during the period 1981-2005 were followed up till the end of 2006 to ascertain their vital status and causes of death. An index of 'relative silicosis effect (RSE)' was used to examine the potential multiplicative interaction between smoking and silicosis. A 
smoking indirectly adjusted standardised morality ratio (SMR) according to the method of 'smoking adjustment factor (SAF)' was presented if the RSE was not statistically significant (i.e., no multiplicative interaction).

Results The RSE for total deaths and the deaths from nonmalignant respiratory diseases (NMRD) was 0.75 (95\% CI: 0.62-0.91) and 0.59 (95\%CI: 0.46-0.78); however, it was not statistically significant for other specific causes of death. Smoking indirectly adjusted SMR for oesophagus cancer, lung cancer, chronic obstructive pulmonary diseases, silicosis, pulmonary tuberculosis and pulmonary heart disease was 1.08 (95\% CI: 0.72-1.64), 1.24 (95\% CI: 1.06-1.45), 2.07 (95\% CI: 1.782.41), 411.35 (95\% CI: 377.03-448.79), 4.99 (95\% CI: $3.80-$ 6.54), and 4.09 (95\% CI: 2.55-6.54).

Conclusions This historical cohort study demonstrated a significant multiplicative interaction between smoking and silicosis on the mortality of total deaths and deaths from NMRD; however, smoking was more likely to play a role of confounding in an increased mortality from other major causes among Chinese silicotic workers.

\section{OCCUPATIONAL EXPOSURE TO TETRACHLOROETHYLENE AND THE RISK OF BLADDER CANCER: A META- ANALYSIS}

1) J Vlaanderen, ${ }^{2}$ Ruder, ${ }^{3}$ Blair, ${ }^{4}$ Hansen, ${ }^{5}$ Charbotel, ${ }^{6}$ Lynge, ${ }^{1}$ Loomis, ${ }^{1} S$ traif, ${ }^{1}$ Guha. ${ }^{1}$ IARC, Lyon, France; ${ }^{2}$ NIOSH, Cincinnati, United States of America; ${ }^{3}$ Division of cancer epidemiology and genetics, national cancer institute, Washington DC, United States of America; ${ }^{4}$ Institute of cancer epidemiology, Danish cancer society, Copenhagen, Denmark; ${ }^{5}$ University Claude Bernard Lyon I, Lyon, France; ${ }^{6}$ Institute of Public Health, Copenhagen, Denmark

\subsection{6/oemed-2013-101717.101}

Objectives The risk of bladder cancer in persons occupationally exposed to tetrachloroethylene was summarised using a metaanalysis.

Methods Studies were identified from a Pubmed literature search including the terms "drycleaner, dry-cleaning, occupation, tetrachloroethylene, bladder cancer, bladder carcinoma, urothelial carcinoma" in various combinations. We included studies that reported a risk estimate specifically for tetrachloroethylene or employment as a "dry cleaner" based on historical information indicating that a large percentage of drycleaners were exposed to tetrachloroethylene but not to other occupational bladder carcinogens. We excluded studies that reported results for "dry-cleaners and laundry workers" (the latter group has not been exposed to tetrachloroethylene), PMR analyses (risk estimates are potentially biassed), and overlapping publications. Publication bias was assessed using funnel plots. All statistical analyses were performed using STATA.

Results Twelve studies were included in the meta-analysis (8 case-control studies, 4 cohort studies) resulting in a meta-RR of 1.24 (95\% CI, 1.12-1.37). The meta-RR was 1.20 (95\% CI, 1.07-1.34) for case-control studies (all adjusted for smoking) and 1.44 (95\% CI, 1.13-1.84) among the cohort studies (none adjusted for smoking). When we restricted the analysis to the studies reporting a risk estimate specifically for exposure to tetrachloroethylene the meta-RR was 1.18 (95\% CI: $1.05-1.33 ; 3$ studies) and for dry cleaners it was 1.46 (95\% CI: 1.17-1.83; 9 studies). A jack knife analysis omitting individual studies demonstrated that there was no overreliance of the overall meta-RR on a single study. There was no evidence of publication bias. One of the included studies assessed exposure-response data and reported an increase in odds ratios with increasing cumulative exposure among men.

Conclusions Occupational exposure to tetrachloroethylene is associated with a moderate, significantly increased risk of bladder cancer. Excesses occurred in cohort and case-control studies. The excesses in case-control studies could not be explained by tobacco use.

\section{OCCUPATIONAL ASBESTOS EXPOSURE AND RISK OF PLEURAL MESOTHELIOMA, LUNG AND LARYNGEAL CANCER IN THE PROSPECTIVE NETHERLANDS COHORT STUDY}

${ }^{1} \mathrm{~N}$ S M Offermans, ${ }^{2}$ Vermeulen, ${ }^{3}$ Burdorf, ${ }^{4}$ Goldbohm, ${ }^{5}$ Kauppinen, ${ }^{2}$ Kromhout, 'van den Brandt. 'Maastricht University Medical Centre, Maastricht, Nederland; ${ }^{2}$ Utrecht University, Utrecht, Nederland; ${ }^{3}$ Erasmus MC, Rotterdam, Nederland; ${ }^{4}$ TNO Quality of Life, Leiden, Nederland; ${ }^{5}$ Finnish Institute of Occupational Health, Helsinki, Finland

\subsection{6/oemed-2013-101717.102}

Objectives Although asbestos research has been ongoing for decades, there are remaining questions regarding cancer risk associated with low exposure and cancer subtypes, the influence of potential confounders, and the interaction between asbestos and smoking. We addressed these questions by studying the association between occupational asbestos exposure and pleural mesothelioma, lung and laryngeal cancer in the prospective population-based Netherlands Cohort Study (NLCS).

Methods The NLCS includes 58279 men aged 55-69 years at enrollment in 1986. Based on job history information obtained from a self-administered questionnaire, asbestos exposure was estimated by linkage to job-exposure matrices. After 17.3 years of follow-up, 132 cases of pleural mesothelioma, 2324 cases of lung cancer, and 166 cases of laryngeal cancer were available for analysis.

Results Overall, occupational asbestos exposure was associated with an increased risk of mesothelioma, lung and laryngeal cancer, also for relatively low exposure. Correcting for potential confounders as age, smoking, alcohol, and several occupational carcinogens hardly influenced these results. Associations with lung cancer subtypes were generally comparable to overall lung cancer, except for adenocarcinoma (HR ever versus never exposed = $1.43,1.52,1.49$ and 0.94 for small cell, large cell, squamous cell and adenocarcinoma respectively). Adenocarcinoma showed only a weak positive association at higher exposure levels for long duration. For laryngeal cancer, associations were usually stronger for supraglottis cancer ( $\mathrm{HR}=2.48,95 \% \mathrm{CI}: 1.33-4.65)$ than glottis cancer ( $\mathrm{HR}=1.12,95 \% \mathrm{CI}: 0.74-1.69)$. There was no statistically significant additive or multiplicative interaction between asbestos and smoking for any of the cancers.

Conclusions The well-established associations between asbestos and mesothelioma, lung and laryngeal cancer were corroborated at relatively low levels of cumulative exposure in the NLCS. Lung adenocarcinoma may only show an increased relative risk at higher asbestos exposure for long duration. Asbestos exposure may be stronger associated with supraglottis cancer than glottis cancer.

103 INTERACTIONS BETWEEN OCCUPATIONAL EXPOSURES
TO EXTREMELY LOW FREQUENCY MAGNETIC FIELD AND
CHEMICALS FOR BRAIN TUMOUR RISK IN THE
INTEROCC STUDY

